\title{
Ventilatory control instability as a predictor of persistent periodic breathing in preterm infants
}

\author{
Leon S. Siriwardhana ${ }^{1}$, Alicia K. Yee ${ }^{1}$, Dwayne L. Mann ${ }^{2,3}$, Shrinkhala Dawadi ${ }^{4}$, Gillian M. Nixon ${ }^{1,5}$, Flora Y. Wong ${ }^{1,6}$, \\ Bradley A. Edwards $s^{2,7,8}$ and Rosemary S. C. Horne ${ }^{1,8}$ 的
}

(c) The Author(s), under exclusive licence to the International Pediatric Research Foundation, Inc 2021

BACKGROUND: Periodic breathing (PB) is common in preterm infants. We aimed to characterize the contribution of ventilatory control instability to the presence and persistence of PB longitudinally.

METHODS: Infants born between 28 and 32 weeks of gestation were studied using daytime polysomnography at: 32-36 weeks postmenstrual age (PMA) $(N=32), 36-40$ weeks PMA $(N=20), 3$ months corrected age $(C A)(N=18)$ and 6 months CA ( $N=19)$. Loop gain, a measure of sensitivity of the ventilatory control system, was estimated by fitting a mathematical model to ventilatory patterns associated with spontaneous sighs.

RESULTS: The time spent in PB decreased from 32-36 weeks PMA to 6 months CA $(P=0.005)$. Across all studies, studies with PB (N $=62)$ were associated with higher loop gain compared to those without $\mathrm{PB}(N=23)$ (estimated marginal mean $\pm \mathrm{SEM}$ : $0.445 \pm 0.01$ vs $0.388 \pm 0.02 ; P=0.020$ ). A threshold of loop gain $>0.415$ (measured at $32-36$ weeks $P M A$ ) provided a sensitivity of $86 \%$ and a specificity of $75 \%$ to detect the presence of PB at 6 months CA.

CONCLUSIONS: The course of PB in preterm infants is related to changes in loop gain. Higher loop gain at 32-36 weeks PMA was associated with a greater risk of persistent PB at 6 months CA.

Pediatric Research (2022) 92:513-519; https://doi.org/10.1038/s41390-021-01816-9

\section{IMPACT:}

- The developmental trajectory of periodic breathing and its relationship to ventilatory control instability is currently unclear.

- Unstable ventilatory control is a determinant of periodic breathing in preterm infants up to 6 months corrected age.

- Infants who display greater ventilatory control instability at 32-36 weeks postmenstrual age may be at increased risk of persistent periodic breathing at 6 months corrected age.

- Assessment of ventilatory control stability may assist in the early identification of infants at risk of persistent periodic breathing and its potential adverse effects.

\section{INTRODUCTION}

Approximately 15 million infants are born preterm $(<37$ weeks of gestational age (GA)) annually worldwide and these numbers are growing. ${ }^{1}$ With improvements in neonatal intensive care techniques, the number of preterm infants surviving at younger gestational ages has increased dramatically. Due to the immaturity of their organs and body systems, preterm infants are at an increased risk of a multitude of short and long-term complications compared to term-born infants. ${ }^{2}$ A common manifestation of immature respiratory control in these infants is that of short repetitive apneas or periodic breathing (PB), ${ }^{3}$ a sleep-related breathing pattern characterized by cyclical apneas of $\sim 4 \mathrm{~s}$ in duration. ${ }^{4,5}$ In preterm infants, PB usually appears in the second week after birth, becomes frequent several weeks after birth ${ }^{6}$, and can be seen after hospital discharge up until 6 months of age or longer. ${ }^{7,8}$ The high prevalence and relatively short duration of apneas associated with PB have led to the notion that PB has no pathological significance. ${ }^{4,9}$ However, reports indicate that PB is a cause of repetitive oxygen desaturation in preterm infants at discharge. ${ }^{10,11}$ Studies have also shown that PB episodes are associated with clinically significant falls in cerebral oxygenation even at 6 months post-term corrected age (CA) in both preterm and term infants, with greater falls in preterm-born infants than those born at term. , $12-14$ In addition, it has been shown that exposure to even short episodes of intermittent hypoxia $(<20 \mathrm{~s})$, similar to that seen with $\mathrm{PB}$, can increase the risk of adverse outcomes during the first three months of life in small for gestational age infants. ${ }^{15}$ Given the evidence from animal studies

\footnotetext{
${ }^{1}$ Department of Paediatrics and The Ritchie Centre, Monash University, Melbourne, VIC, Australia. ${ }^{2}$ Sleep and Circadian Medicine Laboratory, Department of Physiology, Monash University, Melbourne, VIC, Australia. ${ }^{3}$ Institute for Social Science Research, The University of Queensland, Brisbane, QLD, Australia. ${ }^{4}$ Monash Centre for Health Research and Implementation, Monash University, Melbourne, VIC, Australia. ${ }^{5}$ Melbourne Children's Sleep Centre, Monash Children's Hospital, Melbourne, VIC, Australia. ${ }^{6}$ Monash Newborn, Monash Children's Hospital, Melbourne, VIC, Australia. ${ }^{7}$ School of Psychological Sciences and Turner Institute for Brain and Mental Health, Monash University, Melbourne, VIC, Australia. ${ }^{9}$ These authors contributed equally: Bradley A. Edwards, Rosemary S. C. Horne. ${ }^{凶}$ email: rosemary.horne@monash.edu
} 
that indicate that persistent repetitive exposure to hypoxemia can adversely affect neurodevelopment ${ }^{16,17}$ and can alter the development of components of the ventilatory control system with lasting effects, ${ }^{18,19}$ the significance of these short apnea events may need to be reconsidered.

$\mathrm{PB}$ in the preterm infant is likely the result of several interacting mechanisms. Maturational deficits including chemoreceptor hypersensitivity and impaired gas exchange characteristics of the immature lung provide an explanation for the early postnatal destabilization of breathing in the preterm infant. ${ }^{20}$ While a number of pre and postnatal factors such as caffeine therapy have the potential to influence the postnatal trajectory of $\mathrm{PB}^{21}$ the extent to which an innate instability of the ventilatory control system influences the postnatal trajectory of PB is less clear. The engineering concept of loop gain (LG) has been proposed as a tool to quantify the degree of instability of the ventilatory control system. ${ }^{22}$ LG represents the sensitivity of the negative feedback loop that controls ventilation and can be defined as the ratio of the ventilatory response to the disturbance that elicited the response. ${ }^{23} \mathrm{~A}$ high LG represents a hypersensitive ventilatory control system, where a small disturbance leads to a large corrective response, ultimately causing cyclical oscillations in breathing (i.e., PB). ${ }^{23}$ LG can be estimated non-invasively from standard polysomnography (PSG) $)^{24,25}$ and the presence of PB in preterm infants assessed at 36 weeks GA has been associated with elevated LG. ${ }^{26}$ However, what is unclear is (1) how LG relates to the developmental trajectory of PB in preterm infants and (2) whether LG is predictive of the persistence of PB. Accordingly, we aimed to assess the relationship between LG and PB longitudinally in very preterm infants up to 6 months CA. We hypothesized that (1) LG would be related to the presence and the amount of sleep time spent in PB at each time point studied and that (2) a higher LG prior to hospital discharge would predict those infants who had persistent PB at 6 months CA.

\section{METHODS \\ Participants}

Preterm infants born between 28 and 32 weeks of gestation were recruited. Infants were studied longitudinally at four time points: 32-36 weeks postmenstrual age (PMA), 36-40 weeks PMA, 3 and 6 months CA. For inclusion, infants were required to be self-ventilating in room air for at least 3 days prior to the first study. Infants with intrauterine growth restriction, major congenital abnormalities, major intracranial abnormalities (e.g., significant intraventricular hemorrhage [Grade III or IV]), or hemodynamically significant patent ductus arteriosus were excluded. Ethical approval was obtained from the Human Research Ethics Committees of Monash University and Monash Health. Informed written parental consent was obtained for all participants.

\section{Daytime polysomnography}

All studies were conducted during the daytime with $2-4 \mathrm{~h}$ of sleep recorded at each study (see Supplementary Material for a description of the sleep study montage). All infants were studied in the supine position. A trained researcher determined sleep state in $30 \mathrm{~s}$ epochs according to behavioral criteria as active sleep (AS), quiet sleep (QS), or indeterminate sleep (IS). ${ }^{27}$ PB was defined as $\geq 3$ sequential central apneas lasting $\geq 3 \mathrm{~s}$ interrupted by regular breathing lasting $\leq 20 \mathrm{s.}^{28}$ The duration of each PB episode (measured from the start of the first apnea to the end of the last apnea) was used to calculate the \% time spent in PB for each sleep state and for total sleep time (TST). \% TST spent in PB was also categorized as presence ( $>0 \%$ of TST in PB) or absence ( $0 \%$ of TST in PB) of PB and as $<1 \%$ of TST in $\mathrm{PB}$ or $>1 \%$ of TST in $\mathrm{PB}$, based on normative data from term infants. 5,29

\section{Loop gain assessment}

The propensity toward instability of the ventilatory control system (i.e., LG) was calculated by analyzing the transient response to spontaneous sighs that occur frequently during sleep in infants. Movement artifact-free spontaneous sighs were visually identified from the PSG recording which were then imported into MATLAB (R2018a Mathworks Inc., Natick, Massachusetts). A $120 \mathrm{~s}$ analysis window was created, comprising measurements of airflow (linearized nasal pressure trace), respiratory inductance plethysmography traces and oxygen saturation $\left(\mathrm{SpO}_{2}\right), 30 \mathrm{~s}$ preceding and $90 \mathrm{~s}$ following each sigh breath. Estimations of $\mathrm{LG}$ were then derived by fitting a previously validated model of ventilatory control (comprising a gain, time-constant, and delay term) to the pre and post-sigh ventilatory patterns. ${ }^{24,25}$ In the ventilatory control model, the gain term represents the overall $L G$, the "time constant" reflects the time course of the buffering of $\mathrm{CO}_{2}$ in the lung and tissues, and the delay term predominantly reflects the circulation time between the lung and chemoreceptors. The best-matched parameters were then used to transform the ventilatory fluctuations seen in response to a sigh into a ventilatory-drive signal that best matched the observed ventilation (Supplementary Fig. S1). LG was then determined at the natural cycling frequency (i.e., the frequency of PB if breathing was unstable). Individual measures of LG from each window were averaged to obtain an overall value for each infant at each study. Each study required at least one spontaneous sigh in either AS or QS to be included in the analysis.

\section{Statistical analysis}

Statistical analysis was performed using SPSS statistical software (IBM SPSS Statistics version 25, IBM Corp., USA). The differences in the categorical variables over the four studies were assessed using the Chi-square test or Fisher's exact test. Mixed-effects linear regression models were used to explore the association between TST, \% TST spent in AS and QS, \% TST, \% $\mathrm{AS}$, and \% QS spent in PB and LG across PMA. These variables were treated as dependent variables in these models with age studied treated as a fixed effect and participant as a random effect. To assess the relationship between LG and the \% TST spent in PB, age studied and LG were treated as fixed effects and participant as a random effect. LG was treated as the dependent variables in a separate mixed-effects linear regression model to assess whether the presence/absence of PB or $<1 \%$ TST/ $>1 \%$ of TST in PB (treated as fixed effects) were predictive of LG. The models that assessed the changes in \% TST in PB had non-normally distributed residuals. To account for this, a sensitivity check was conducted by square-root transforming the dependent variables in these models which yielded similar results. Therefore, the original non-transformed model results are presented. Receiver operating characteristic (ROC) analyses were used to determine optimum thresholds for LG (based on sensitivity and specificity) that predicted the presence/absence of PB at 6 months CA.

Descriptive data are presented as median [interquartile range (IQR)] or number $(\% \mathrm{~N})$ as appropriate. All data from mixed-effects linear regression models are presented as estimated marginal means $(E M M) \pm$ standard error of the mean (SEM) or as estimated coefficient and standard error (SE). $P$-values were adjusted for multiple comparisons with Bonferroni correction for the regression models. $P<0.05$ was considered statistically significant.

\section{RESULTS}

A total of 32 infants ( $19 \mathrm{~F} / 13 \mathrm{M}$ ) were recruited. Infants had a median [IQR] GA at birth of $31[30,32]$ weeks, birthweight of 1426 [1307, $1614] \mathrm{g}$, and APGAR scores of $7[6,9]$ at $1 \mathrm{~min}$ and $9[7,9]$ at $5 \mathrm{~min}$. All infants had been administered caffeine for apnea of prematurity during their hospital stay and 59\% were still on caffeine treatment at the first study. All 32 infants were studied at 32-36 weeks PMA, 20 infants at 36-40 weeks PMA, 18 infants at 3 months CA and 19 infants at 6 months $C A$ (Fig. 1). Of note, three infants were studied at 7 months CA (instead of 6) due to COVID-19 (Coronavirus disease of 2019)-related restrictions. Study-specific demographic and sleep characteristics are shown in Table 1. TST decreased, the \% time spent in AS decreased and the \% time spent in QS increased with increasing PMA as expected. Due to the relatively small amount of time spent in IS $(<1 \%$ of TST), this was combined with AS (Table 1$)$.

\section{Periodic breathing episodes}

The \% TST spent in PB sharply decreased from 32 to 36 weeks PMA to 6 months CA (Table 1). \% time spent in PB in both AS and QS showed similar trends to \% TST spent in PB, with slightly greater time spent in $\mathrm{PB}$ in AS compared to $\mathrm{QS}$ at 32-36 weeks PMA, 36-40 weeks PMA and 3 months CA. At 6 months CA, 7/19 


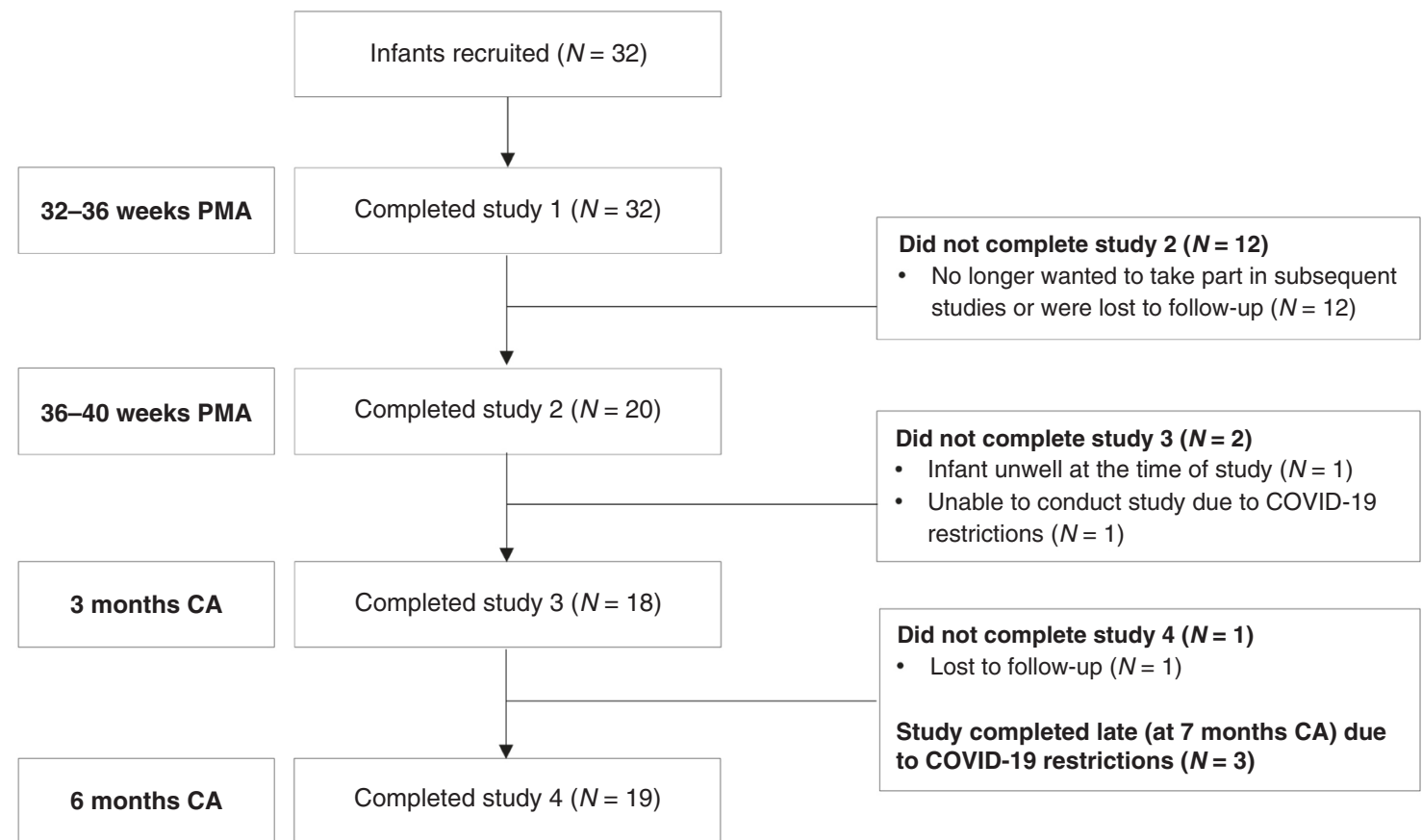

Fig. 1 Flow diagram demonstrating infants included in the analysis at each time point. All 32 infants were studied at 32-36 weeks PMA. 20 infants were studied at 36-40 weeks PMA, 12 infants were lost to follow-up. 18 infants completed the study at 3 months CA with 2 infants unable to complete the study. 19 infants completed the study at 6 months CA, 1 infant was lost to follow-up. 16 infants completed all four studies. PMA postmenstrual age, CA corrected age, COVID-19 Coronavirus disease of 2019.

Table 1. Study-specific demographic, sleep, periodic breathing, and sigh characteristics.

\begin{tabular}{|c|c|c|c|c|c|}
\hline & $\begin{array}{l}\text { Study } 1 \\
\text { 32-36 weeks PMA }\end{array}$ & $\begin{array}{l}\text { Study } 2 \\
36-40 \text { weeks PMA }\end{array}$ & $\begin{array}{l}\text { Study } 3 \\
3 \text { months CA }\end{array}$ & $\begin{array}{l}\text { Study } 4 \\
6 \text { months CA }\end{array}$ & $P$-value \\
\hline$N$ & 32 & 20 & 18 & 19 & - \\
\hline PMA (weeks) & $34[33,35]$ & $39[38,40]$ & $\begin{array}{l}53 \\
{[53,54]}\end{array}$ & $\begin{array}{l}67 \\
{[66,69]}\end{array}$ & - \\
\hline $\operatorname{Sex}(\% \mathrm{~F})$ & 59 & 60 & 61 & 63 & 0.077 \\
\hline \multicolumn{6}{|l|}{ Sleep indices } \\
\hline$\%$ AS & $58 \pm 2$ & $58 \pm 2$ & $48 \pm 3$ & $42 \pm 4$ & 0.003 \\
\hline \multicolumn{6}{|l|}{ Periodic breathing indices } \\
\hline$\%$ PB TST & $13 \pm 3$ & $9 \pm 2$ & $1 \pm 1^{\mathrm{a}, \mathrm{b}}$ & $3 \pm 1^{\mathrm{a}}$ & 0.001 \\
\hline$\%$ PB QS & $11 \pm 3$ & $6 \pm 1$ & $1 \pm 1^{\mathrm{a}, \mathrm{b}}$ & $3 \pm 2$ & $<0.001$ \\
\hline$\%$ PB AS & $14 \pm 3$ & $10 \pm 3$ & $2 \pm 1^{a, b}$ & $2 \pm 1^{\mathrm{a}}$ & 0.023 \\
\hline Analyzable sighs per study & $8 \pm 1$ & $10 \pm 1$ & $8 \pm 1$ & $6 \pm 1$ & 0.078 \\
\hline Number of infants with analyzable sighs in TST $(\% N)$ & $31(97)$ & $20(100)$ & $18(100)$ & $17(89)$ & 0.229 \\
\hline Number of infants with analyzable sighs in QS (\% $N)$ & $30(94)$ & $18(90)$ & $18(100)$ & $16(84)$ & 0.331 \\
\hline Number of infants with analyzable sighs in AS $(\% N)$ & $22(69)$ & $14(70)$ & $12(67)$ & $11(58)$ & 0.847 \\
\hline
\end{tabular}

Data from indeterminate sleep (IS) has been combined with AS data. Data are presented as median [interquartile range], number (\% $N$ ) or as estimated marginal means \pm standard error of the mean. Data presented as $\% N$ and $\% \mathrm{~F}$ were assessed using Chi-Square or Fisher's exact tests. Data presented as estimated marginal means \pm standard error of the mean were assessed using mixed-effects linear regression models.

$F$ female, PMA postmenstrual age, CA corrected age, TST total sleep time, AS active sleep, QS quiet sleep, PB periodic breathing.

${ }^{\text {a }} P<0.05$ statistically significant versus study 1 .

${ }^{\mathrm{b}} P<0.05$ statistically significant versus study 2 . 

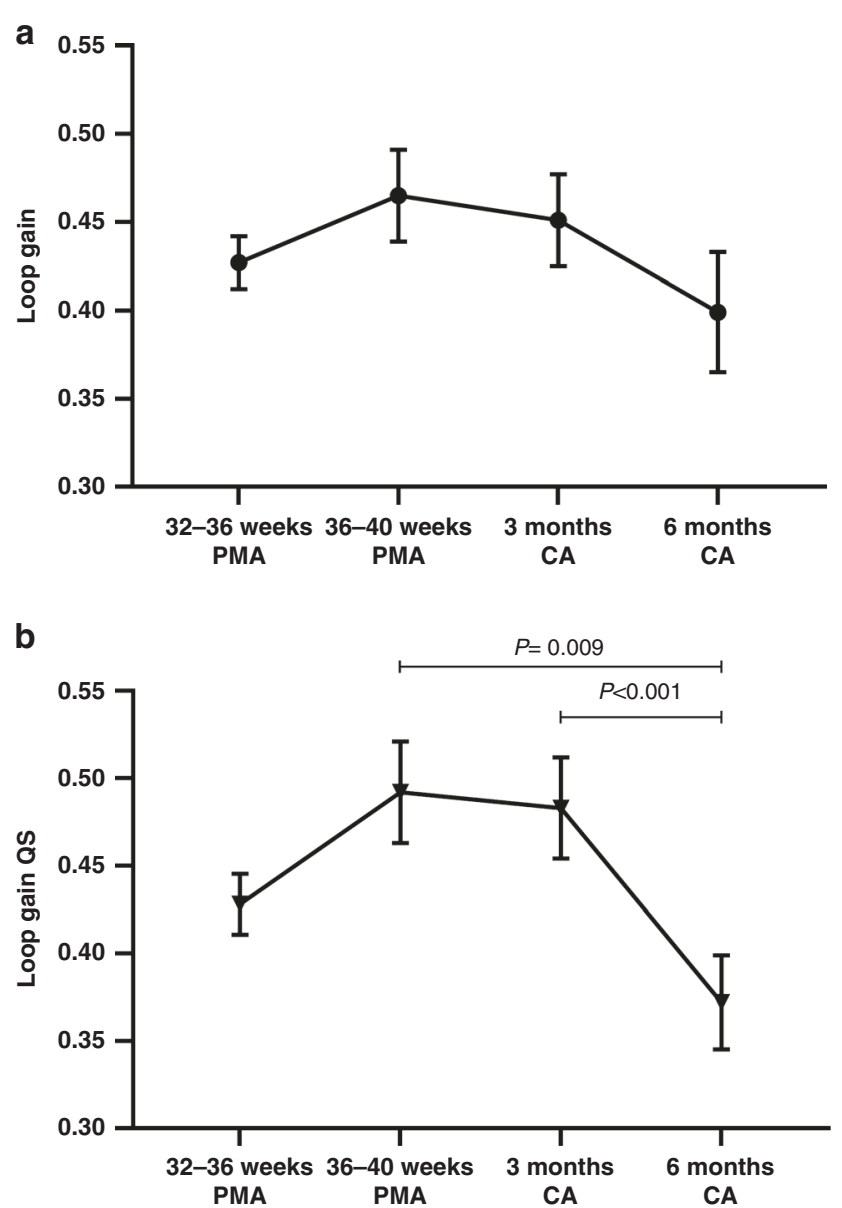

Fig. 2 Loop gain over the time points studied. a Loop gain measured from sigh events across both sleep states. b Loop gain measured from sigh events in quiet sleep only. Data presented as estimated marginal means and standard error of mean obtained from the linear mixed model with time studied treated as a fixed effect and participant treated as a random effect. QS quiet sleep, PMA postmenstrual age, CA corrected age.

(37\%) displayed at least one episode of PB at the time of the study.

\section{Loop gain}

Most infants had 6-10 analyzable sighs per study across TST to calculate LG at each time point studied (Table 1). LG measured across TST decreased with increasing PMA from 36 to 40 weeks PMA, although this did not reach statistical significance between studies (Fig. 2a). However, LG measured in QS significantly decreased from 36 to 40 weeks PMA to 6 months CA and from 3 months CA to 6 months (Fig. 2b). Due to the reduced number of infants at each time point with artifact-free sighs in AS (Table 1), LG was not assessed separately in AS across PMA. At Study 1, there was no difference in LG between infants on caffeine treatment $(N=19)$ compared to those who were not $(N=13)(0.42[0.37$, $0.45]$ vs $0.36[0.35,0.55] ; P=0.921$ ) (assessed using Mann-Whitney $U$ test). In addition, there was no association between LG and mean oxygen saturation across all the sigh windows analyzed, regardless of the time studied $(r=0.043 ; P=0.695)$ (assessed using Spearman correlation).

\section{Relationship between loop gain and periodic breathing indices}

Regression analysis showed that both age studied $(P<0.001)$ and LG $(P=0.005)$ significantly predicted the change in the \% TST spent in
PB. LG (measured in total sleep) was positively related to \% time spent in PB (estimated coefficient: 9.29, SE: 2.59). When this model was repeated using LG measured only in $Q S$, a stronger association was found (estimated coefficient: 6.42, SE: 1.45, $P=0.001$ ).

Similarly, regression analysis showed that across all studies, the presence/absence of PB was a significant predictor of LG where studies with PB $(N=62)$ were associated with higher LG compared to those studies without $\mathrm{PB}(N=23)(0.44 \pm 0.01$ vs $0.39 \pm 0.02$; $P=0.020)$. Studies with $>1 \%$ PB TST $(N=48)$ were also associated with higher LG compared to those with $<1 \%$ PB TST $(N=39)(0.46$ \pm 0.11 vs $0.40 \pm 0.11 ; P=0.014$ ).

ROC analysis showed that when infants who were studied late due to COVID-19 restrictions were excluded $(N=3)$, LG measured at 32-36 weeks PMA (Study 1), predicted the presence/absence of PB at 6 months CA (infants with PB $N=7$, infants without PB $N=$ 8) (Fig. 3a). A threshold of $L G>0.415$ provided a sensitivity of $86 \%$, specificity of $75 \%$, positive predictive value (PPV) of $75 \%$, and a negative predictive value (NPV) of $86 \%$ to detect the presence of $\mathrm{PB}$ at 6 months. This relationship was stronger when LG measured in QS at Study 1 was used (infants with PB $N=7$, infants without PB $N=7$ ) (Fig. 3b), where LG $>0.420$ provided a sensitivity of $86 \%$, specificity of $86 \%$, PPV of $86 \%$ and a NPV of $86 \%$ to detect the presence of PB. Of note, the \% TST spent in PB at Study 1 was not predictive of the presence/absence of $P B$ at 6 months CA (area under the curve: $0.698 ; P=0.186$ ).

\section{DISCUSSION}

This study provides new insights into the developmental trajectory of PB, using ventilatory control instability assessed by LG as an explanatory factor. We identified that the overall change in \% TST spent in PB with increasing PMA was associated with LG, and that the presence of PB was associated with higher $L G$, regardless of the age studied. In addition, $L G$ measured at 32-36 weeks PMA, prior to discharge from the neonatal unit, appears to be a moderately sensitive and specific measure that can identify infants at increased risk of persistent $P B$ at 6 months CA.

To our knowledge, this is the first study to report the developmental trajectory of LG in preterm infants. The reductions in the \% TST spent in PB observed with increasing PMA in our study are similar to previous reports that have assessed PB longitudinally in preterm infants after hospital discharge. ${ }^{4,7,8,12}$ LG showed a corresponding decline indicating greater stabilization at 6 months CA compared to 36-40 weeks PMA, particularly when measured in $\mathrm{QS}$, highlighting its role in the developmental trajectory of PB. There is a paucity of literature assessing the developmental trajectory of ventilatory control in infants, although it is reported to reach stable adult levels by 6 months postnatal age in term infants. ${ }^{20,30}$ It is thought that this time period coincides with maturational effects of the carotid body leading to a reduction in the response time of the peripheral chemoreceptors. ${ }^{31}$ This may also provide an explanation for our observation of a stronger relationship in QS, a sleep state in which chemical control of breathing predominates. ${ }^{32}$ However, in the preterm infant, several other factors are likely to affect the development of ventilatory control, including the degree of prematurity, presence of comorbidities (e.g., chronic neonatal lung disease), and environmental exposures (e.g., oxygenation profiles) in the early neonatal period. ${ }^{33}$ Despite the degree of prematurity and comorbidities being controlled for in this study, individual variation in factors such as functional residual capacity ${ }^{34,35}$ and varying levels of exposure to hypoxia and hyperoxia ${ }^{36}$ are likely to provide an explanation for the high inter-individual variation in the trajectory of LG.

Our findings that LG was higher in studies with PB compared to those without, regardless of the age studied, are supported by a previous study which assessed preterm infants born between 


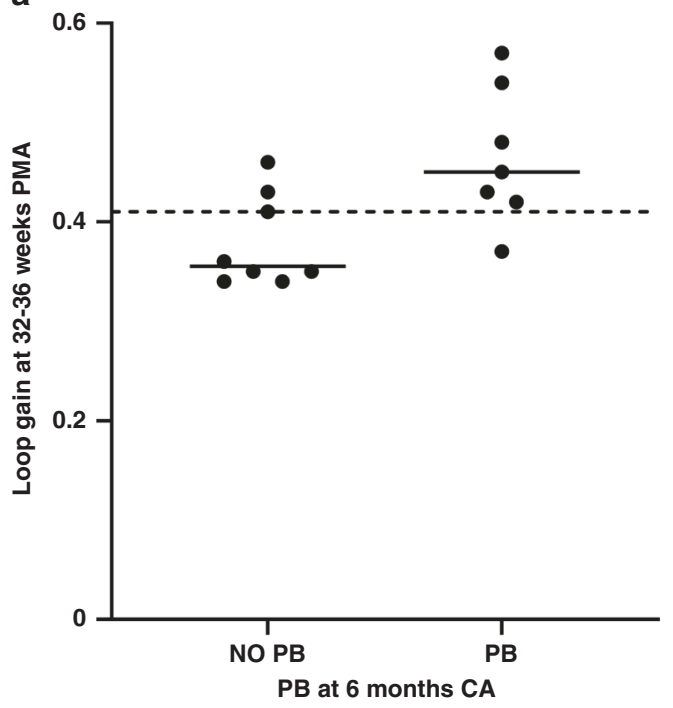

b

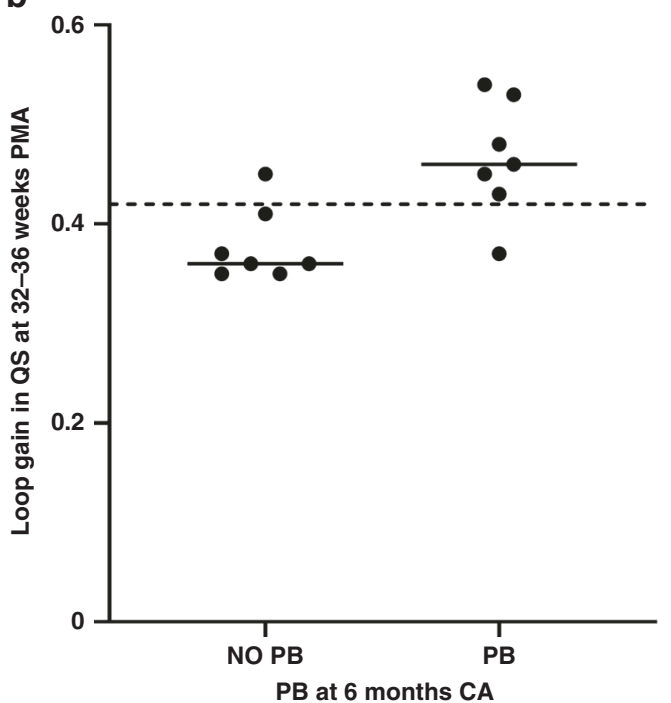

$\operatorname{AUC}(95 \% \mathrm{Cl}) \quad 0.866(0.682-1.000)$ $P$ value $\quad 0.018$

Sensitivity $\quad 86 \%$ PPV $75 \%$

Specificity $\quad 75 \% \quad$ NPV $86 \%$

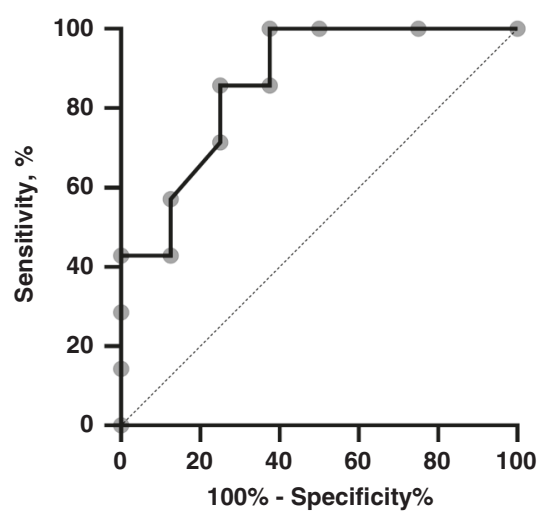

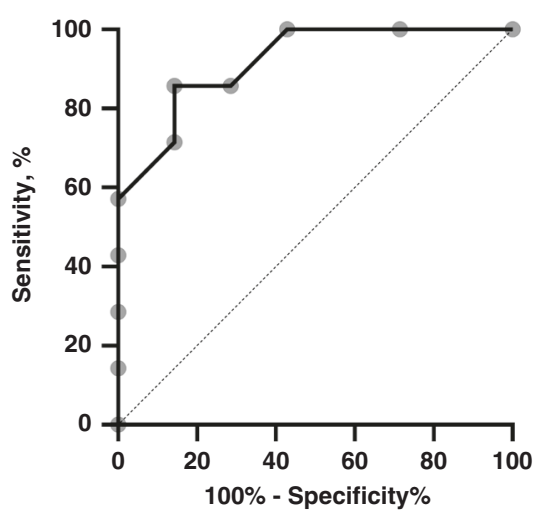

Fig. 3 Receiver operating characteristic (ROC) analysis of loop gain measured at 32-36 weeks PMA as a predictor of persistent PB. a ROC analysis for loop gain measured in both sleep states at 32-36 weeks PMA to predict the presence ( $>0 \%$ of TST in PB) or absence (0\% of TST in $\mathrm{PB}$ ) of PB at 6 months $\mathrm{CA}$ (infants with PB $N=7$, infants without PB $N=8$ ). b ROC analysis for loop gain measured in QS at 32-36 weeks PMA to the predict presence ( $>0 \%$ of TST in PB) or absence ( $0 \%$ of TST in PB) of PB at 6 months CA (infants with PB $N=7$, infants without PB $N=7$ ). Infants studied past 6 months CA were excluded for both analyses $(N=3)$. PB periodic breathing, PMA postmenstrual age, $C A$ corrected age, AUC area under the curve, PPV positive predictive value, NPV negative predictive value.

24 and 28 weeks of gestation and studied at 36 weeks PMA. ${ }^{26}$ Despite only being measured at one time point, the authors also identified that LG measured in QS was predictive of the presence or absence of $\mathrm{PB}^{26} \mathrm{~A}$ novel finding of our study was that LG measured at 32-36 weeks PMA could also be useful in predicting infants likely to have persistent PB at 6 months CA. Growing evidence indicates that $\mathrm{PB}$ is associated with falls in cerebral oxygenation in infants born preterm ${ }^{8,12}$ and prolonged exposure to intermittent hypoxia has the potential to adversely affect neurodevelopmental outcomes. ${ }^{36,37}$ In addition, emerging evidence suggests that children born preterm have a phenotype of altered ventilatory control compared to term-born children that increases their risk of developing other sleep-related breathing instabilities, such as obstructive sleep apnea, later in life. ${ }^{38-40}$ Therefore, future studies are needed to confirm whether LG is a useful tool to identify infants who could be at greater risk of these adverse outcomes, prior to discharge from the neonatal unit.

\section{Methodological considerations}

There are several limitations that need to be considered in the interpretation of our findings. First, 35\% of infants were lost to follow-up, which reduced our sample size at studies after hospital discharge. However, we were able to increase the robustness of the available data with the application of a linear mixed model analysis. Second, studies had to be conducted to coincide with the infant's usual daytime sleep, and to reduce parent inconvenience of returning to the hospital on three occasions. Thus, it is possible that our recording time was not sufficient to capture the full extent of $\mathrm{PB}$ that might be present during overnight sleep, which may have led to either an over or underestimation of the presence of PB at each age. However, the amounts of time spent in PB were similar to that reported previously in studies of longer duration. ${ }^{4}$ Third, $59 \%$ of infants were on caffeine therapy at the time of the first study. The effect of caffeine treatment on LG is currently unclear, however, our sensitivity analysis revealed no effect of caffeine on LG at Study 1. 
Lastly, our estimate of LG assumes that spontaneous sighs occurred as an external disturbance to the ventilatory control system, however, this is supported by existing literature. ${ }^{41}$

\section{CONCLUSIONS}

For the first time, we describe the relationship between ventilatory control instability assessed using LG and the amount of sleep time spent in PB in preterm infants longitudinally up to 6 months CA. LG has moderate sensitivity and specificity for detecting infants at increased risk of persistent PB. Future studies that extend these findings to assess the ability of LG to predict PB episodes with clinically significant falls in oxygen saturation or cerebral oxygenation are warranted.

\section{REFERENCES}

1. Walani, S. R. Global burden of preterm birth. Int. J. Gynaecol. Obstet. 150, 31-33 (2020).

2. Eichenwald, E. C. \& Stark, A. R. Management and outcomes of very low birth weight. N. Engl. J. Med. 358, 1700-1711 (2008).

3. Fairchild, K. et al. Clinical associations of immature breathing in preterm infants: part 1-central apnea. Pediatr. Res. 80, 21-27 (2016).

4. Glotzbach, S. F., Baldwin, R. B., Lederer, N. E., Tansey, P. A. \& Ariagno, R. L. Periodic breathing in preterm infants: incidence and characteristics. Pediatrics 84, 785-792 (1989).

5. Kelly, D. H., Stellwagen, L. M., Kaitz, E. \& Shannon, D. C. Apnea and periodic breathing in normal full-term infants during the first twelve months. Pediatr. Pulmonol. 1, 215-219 (1985).

6. Patel, M. et al. Clinical associations with immature breathing in preterm infants: part 2-periodic breathing. Pediatr. Res. 80, 28-34 (2016).

7. Albani, M., Bentele, K. H., Budde, C. \& Schulte, F. J. Infant sleep apnea profile: preterm vs. term infants. Eur. J. Pediatr. 143, 261-268 (1985).

8. Horne, R. S. C. et al. Comparison of the longitudinal effects of persistent periodic breathing and apnoea on cerebral oxygenation in term- and preterm-born infants. J. Physiol. 596, 6021-6031 (2018).

9. Hodgman, J. E., Gonzalez, F., Hoppenbrouwers, T. \& Cabal, L. A. Apnea, transient episodes of bradycardia, and periodic breathing in preterm infants. Am. J. Dis. Child. 144, 54-57 (1990).

10. Poets, C. F. \& Southall, D. P. Patterns of oxygenation during periodic breathing in preterm infants. Early Hum. Dev. 26, 1-12 (1991).

11. Razi, N. M., DeLauter, M. \& Pandit, P. B. Periodic breathing and oxygen saturation in preterm infants at discharge. J. Perinatol. 22, 442-444 (2002).

12. Decima, P. F., Fyfe, K. L., Odoi, A., Wong, F. Y. \& Horne, R. S. The longitudinal effects of persistent periodic breathing on cerebral oxygenation in preterm infants. Sleep. Med. 16, 729-735 (2015).

13. Horne, R. S. C. et al. The longitudinal effects of persistent apnea on cerebral oxygenation in infants born preterm. J. Pediatr. 182, 79-84 (2017).

14. Jenni, O. G. et al. Cyclical variations in cerebral blood volume during periodic breathing. Acta Paediatr. 83, 1095-1096 (1994).

15. Di Fiore, J. M. et al. Patterns of oxygenation, mortality, and growth status in the surfactant positive pressure and oxygen trial cohort. J. Pediatr. 186, 49-56 (2017). e41.

16. Darnall, R. A. et al. Early postnatal exposure to intermittent hypoxia in rodents is proinflammatory, impairs white matter integrity, and alters brain metabolism. Pediatr. Res. 82, 164-172 (2017).

17. Di Fiore, J. M. \& Raffay, T. M. The relationship between intermittent hypoxemia events and neural outcomes in neonates. Exp. Neurol. 342, 113753 (2021).

18. Pawar, A., Peng, Y. J., Jacono, F. J. \& Prabhakar, N. R. Comparative analysis of neonatal and adult rat carotid body responses to chronic intermittent hypoxia. J. Appl. Physiol. 104, 1287-1294 (2008).

19. Peng, Y. J., Rennison, J. \& Prabhakar, N. R. Intermittent hypoxia augments carotid body and ventilatory response to hypoxia in neonatal rat pups. J. Appl. Physiol. 97, 2020-2025 (2004).

20. Edwards, B. A., Sands, S. A. \& Berger, P. J. Postnatal maturation of breathing stability and loop gain: the role of carotid chemoreceptor development. Respir. Physiol. Neurobiol. 185, 144-155 (2013).

21. Seppä-Moilanen, M., Andersson, S., Rantakari, K., Mikkola, K. \& Kirjavainen, T. Caffeine and supplemental oxygen effectively suppress periodic breathing with only minor effects during long episodes of apnoea in preterm infants. Acta Paediatr. 108, 443-451 (2019).

22. Khoo, M. C., Kronauer, R. E., Strohl, K. P. \& Slutsky, A. S. Factors inducing periodic breathing in humans: a general model. J. Appl. Physiol. 53, 644-659 (1982).

23. Younes, M. The physiologic basis of central apnea and periodic breathing. Curr. Pulmonol. 10, 265-326 (1989).
24. Nava-Guerra, L. et al. Quantifying ventilatory control stability from spontaneous sigh responses during sleep: a comparison of two approaches. Physiol. Meas. 39, 114005 (2018).

25. Terrill, P. I. et al. Quantifying the ventilatory control contribution to sleep apnoea using polysomnography. Eur. Respir. J. 45, 408-418 (2015).

26. Edwards, B. A. et al. Assessing ventilatory instability using the response to spontaneous sighs during sleep in preterm infants. Sleep 41, zsy161 (2018).

27. Curzi-Dascalova, L. \& Mirmiran, M. Manual of Methods for Recording and Analyzing Sleep-Wakefulness States in Preterm and Full-Term Infant (Les Editions INSERM, Paris, 1996).

28. Berry, R. B. et al. AASM scoring manual updates for 2017 (version 2.4). J. Clin. Sleep. Med. 13, 665-666 (2017).

29. Brockmann, P. E., Poets, A. \& Poets, C. F. Reference values for respiratory events in overnight polygraphy from infants aged 1 and 3months. Sleep. Med. 14, 1323-1327 (2013).

30. Fleming, P. J., Goncalves, A. L., Levine, M. R. \& Woollard, S. The development of stability of respiration in human infants: changes in ventilatory responses to spontaneous sighs. J. Physiol. 347, 1-16 (1984).

31. Wilkinson, M. H. et al. Postnatal development of periodic breathing cycle duration in term and preterm infants. Pediatr. Res. 62, 331-336 (2007).

32. Phillipson, E. A. Control of breathing during sleep. Am. Rev. Respir. Dis. 118, 909-939 (1978).

33. Carroll, J. L. Developmental plasticity in respiratory control. J. Appl. Physiol. 94, 375-389 (2003).

34. Poets, C. F., Rau, G. A., Neuber, K., Gappa, M. \& Seidenberg, J. Determinants of lung volume in spontaneously breathing preterm infants. Am. J. Respir. Crit. Care Med. 155, 649-653 (1997).

35. Tourneux, P. et al. Relationship between functional residual capacity and oxygen desaturation during short central apneic events during sleep in "late preterm" infants. Pediatr. Res. 64, 171-176 (2008).

36. Bavis, R. W. Developmental plasticity of the hypoxic ventilatory response after perinatal hyperoxia and hypoxia. Respir. Physiol. Neurobiol. 149, 287-299 (2005).

37. Poets, C. F. et al. Association between intermittent hypoxemia or bradycardia and late death or disability in extremely preterm infants. JAMA 314, 595-603 (2015).

38. Armoni Domany, K. et al. Cardioventilatory control in preterm-born children and the risk of obstructive sleep apnea. Am. J. Respir. Crit. Care Med. 197, 1596-1603 (2018).

39. Raynes-Greenow, C. H. et al. Sleep apnea in early childhood associated with preterm birth but not small for gestational age: a population-based record linkage study. Sleep 35, 1475-1480 (2012).

40. Rosen, C. L. et al. Prevalence and risk factors for sleep-disordered breathing in 8to 11-year-old children: association with race and prematurity. J. Pediatr. 142, 383-389 (2003).

41. Qureshi, M., Khalil, M., Kwiatkowski, K. \& Alvaro, R. E. Morphology of sighs and their role in the control of breathing in preterm infants, term infants and adults. Neonatology 96, 43-49 (2009).

\section{ACKNOWLEDGEMENTS}

We would like to thank Ms. Emma Yeomans and Ms. Kristy Elsayed for their assistance with data collection in Monash Newborn and Dr. Eldho Paul and Dr. Shane Landry for their assistance with statistical analysis. We would also like to thank all the parents and their babies who participated in the study and the staff of Monash Newborn and the Melbourne Children's Sleep Centre, where the studies were carried out.

\section{AUTHOR CONTRIBUTIONS}

L.S.S. carried out the data analyses, drafted the initial manuscript, and revised the manuscript for important intellectual content. A.K.Y. and D.L.M. assisted with the data analyses and reviewed and revised the manuscript for important intellectual content. S.D. assisted with the statistical analysis and reviewed and revised the manuscript for important intellectual content. G.M.N. conceptualized and designed the study, obtained funding, and reviewed and revised the manuscript for important intellectual content. F.Y.W. conceptualized and designed the study, obtained funding, coordinated data collection, and reviewed and revised the manuscript for important intellectual content. B.A.E. supervised data collection and analysis, and reviewed and revised the manuscript for important intellectual content. R.S.C.H. conceptualized and designed the study, obtained funding, coordinated and supervised data collection and analysis, and reviewed and revised the manuscript for important intellectual content.

\section{FUNDING}

This project was supported by funding from the Scottish Cot Death Trust and the Rebecca Cooper Foundation. Mr. Siriwardhana was supported by the Australian 
Government's Research Training Program Stipend. B.A.E. is supported by a Heart Foundation of Australia Future Leader Fellowship (101167). R.S.C.H. is supported by a National Health and Medical Research Council of Australia Investigator Grant (1195453).

\section{COMPETING INTERESTS}

The authors declare no competing interests.

\section{CONSENT STATEMENT}

Informed written consent was obtained from the parent and/or legal guardian of the infant prior to inclusion in the study.

\section{ADDITIONAL INFORMATION}

Supplementary information The online version contains supplementary material available at https://doi.org/10.1038/s41390-021-01816-9.

Correspondence and requests for materials should be addressed to Rosemary S. C. Horne.

Reprints and permission information is available at http://www.nature.com/ reprints

Publisher's note Springer Nature remains neutral with regard to jurisdictional claims in published maps and institutional affiliations. 